\title{
Current Issues in Cigarette Smoking Among Persons Living with HIV/AIDS: A Growing Public Health Problem Surrounded by Missing Information and Misconceptions
}

\section{Maria Jose Miguez*}

Department of Epidemiology and Public Health, Florida International University, USA

\section{The Tobacco Epidemic}

According to the World Health Organization (WHO), tobacco is one of the world's leading risk factors for premature morbidity and mortality. [1] More people die each year from tobacco related diseases than from HIV, car accidents, alcohol use, illegal drug use, murders, and suicides combined [1]. Worldwide, over two million deaths are attributable to cigarette smoking and estimates indicated that tobacco will kill one billion people worldwide in the $21^{\text {st }}$ century [2].

In the United States, approximately 45.3 million American adults age 18 and older are current cigarette smokers. However, rates of smoking widely varied from $9.8 \%$ in Utah to $28.6 \%$ in Kentucky [2]. The magnitude of the problem is even bigger among people living with HIV (PLWH) with skyrocketing rates ranging from 40 to $75 \%$ [3-5]. From an epidemiologic perspective almost 1.2 million persons in the U.S. are living with HIV/AIDS, and approximately 40,000 new cases are diagnosed each year [6]. With the extended survival achieved with highly active antiretroviral therapy (HAART), this means that there will be an ever growing population living both with HIV/AIDS and tobacco-related complications. These statistics are enough to make you scratch your head in wonderment why they have not deserve special attention.

In addition, now we need to embrace HIV as a chronic disease, in which emerging non-AIDS conditions such as cardiovascular (CVD), chronic kidney (CKD) and central nervous system (CNS) diseases, will need to be addressed [7-8]. Under these conditions smoking will become highly relevant. Although the link between cigarette smoking and disease has been well-characterized in non-HIV infected populations [1-2], evidence for the influence of smoking on HIV disease is limited and inconclusive. First, compared to the extensive literature on drugs of abuse, little research has been specifically devoted to the investigation of tobacco's effects [9-10]. For example, the review by Furber et al. in 2006 identified only 10 studies using progression to AIDS as an end point, nine of which found no relation with tobacco smoking [9]. In 2009, Marshall et al. identified only sixteen prospective cohort or case-control studies evaluating the relationship between cigarette smoking and HIV progression, with a variety of endpoints, including CD4 cell counts, time to AIDS, incidence of AIDS, and incidence of opportunistic infections, or other HIV-related clinical events (four of them were published by our group) [10]. Both reviews concluded that there is conflicting data on whether cigarette smoking is associated with HIV progression, and highlights the need of additional studies.

Discrepancies may be attributed to several factors: First, many of those initial studies were performed in the pre-ART era, when HIV was a lethal disease. The variation in populations explored by these studies (e.g., studies with mostly men, MSM, or injection drug users) may explain differences in results. In addition, there is a pervasive lack of functional studies, as researchers and clinicians have been mostly concentrated in assessing quantitative and not in functional assessments.

\section{Cigarette Smoking in Immunity and Inflammation}

Since the beginning of the HIV epidemic, CD4 cell counts were selected as a clinical marker of HIV, although it was clearly established that functional defects of immune cells were present in PLWH [11]. Nevertheless, given that functional markers were cumbersome and expensive, CD4 cell counts were selected as a research and clinical tool. [11] Unfortunately, the pervasive lack of functional studies has created the erroneous impression that tobacco is somehow safe. Furthermore, since lipopolysaccharide (LPS) and the tobacco-related protein (TGP) stimulate peripheral T-cell proliferation, this "polyclonal" expansion of $\mathrm{T}$ cells have resulted in the dangerous idea that tobacco may be even beneficial [12]. In addition, one can expect that with the extended survival times, the deleterious effects of cigarette smoking on the immune system will become more evident. Recently, the Women's Health Initiative [13] has clearly indicated that smokers who initiated ART have a lower chance of achieving a viral (hazard ratio $(\mathrm{HR})=0.79$; $\mathrm{P}=0.006)$ or immunologic response $(\mathrm{HR}=0.85 ; \mathrm{P}=0.041)$, and a greater chance of developing viral $(\mathrm{HR}=1.39 ; \mathrm{P}=0.013)$ or immunologic failure $(\mathrm{HR}=1.52 ; \mathrm{P}=0.001)$, or dying $(\mathrm{HR}=1.53 ; \mathrm{P}=0.018)$.

Despite the groundbreaking biomedical advances of HAART in controlling viral load, HAART has failed to reduce inflammatory markers to normal levels. This is of great concern given that studies have consistently demonstrated that $\mathrm{CI}$ increases oxidative stress, and disrupts tissue architecture, leading to impaired end-organ function and immune exhaustion, with the resulting higher risk for AIDS and nonAIDS related diseases. Despite extensive efforts [14-21], the triggers of CI have yet to be fully established in order to prevent premature morbidity and mortality. Poor adherence sub-optimal immune recovery and viral co-infections (i.e., $\mathrm{HB}, \mathrm{CMV}$ ) have been identified as important predictors of CI [14-21]. Noteworthy, those previous studies either failed to analyze the potential effects of smoking on the chronic inflammatory response, or used stored serum/plasma which is not ideal samples [14-21]. In this regard, analyses from our ongoing studies, using supernatants of stimulated mononuclear cells, revealed that in smokers receiving HAART inflammatory markers remain high (IL-6, TNF- $\alpha$ ), compared to socio-demographically matched people

*Corresponding author: Maria Jose Miguez, Associate Professor, Department of Epidemiology and Public Health, Florida International University, USA, Tel: 7864273054; E-mail: marisemp01@att.net

Received August 27, 2012; Accepted August 28, 2012; Published August 30 2012

Citation: Miguez MJ (2012) Current Issues in Cigarette Smoking Among Persons Living with HIVIAIDS: A Growing Public Health Problem Surrounded by Missing Information and Misconceptions. J AIDS Clinic Res 3:e109. doi:10.4172/2155$6113.1000 \mathrm{e} 109$

Copyright: (c) 2012 Miguez MJ. This is an open-access article distributed unde the terms of the Creative Commons Attribution License, which permits unrestricted use, distribution, and reproduction in any medium, provided the original author and source are credited. 
without this habit. Notably, increases were more marked among users of mentholated cigarette [22]. This finding warrants attention, given the physiopathological consequences of CI.

In this context, is clearly evident that the effects of tobacco need to be re-evaluated in this new HAART era and the plausible effects of tobacco plus menthol further explore. It also highlights the relevance that health care providers and stakeholders should focus on prevalent problems such as smoking that have been placed behind in the prevention priority lists.

\section{Nicotine and HIV-Associated Dementia}

Despite successful viral control and CD4 replenishment, morbidity and mortality rates are overly high among PLWH [23-24]. While AIDSrelated conditions have been significantly reduced, other diseases have emerged as potential threats to survival and quality of life, including, but not limited to cardiovascular and central nervous system illnesses. According to a CHARTER [23] report, prevalence can be above $50 \%$, although perhaps mostly in their milder forms. These findings are of great concern because cognitive deficits may impair a person's capability to adhere to complex medical regimens, but of utmost importance, cognitive impairment has been related to reduced quality of life and survival [23-24]. Although the pathogenic mechanisms behind the central nervous system (CNS) dysfunction in the pre-ART era remain clear to a certain extent, many of them are no longer valid. Moreover, the steady rates of HIV associated neurological Disorders (HAND) clearly indicate that there are additional underlying risk factors that need to be identified. Therefore, what factors are prevalent enough and biologically plausible to contribute significantly to these long-term adverse outcomes in PLWH receiving effective ART?

As in other health areas, there is insufficient data on the neurocognitive effects of tobacco among PLWH. Prior to ART, Burns et al. demonstrated an increased risk of developing HIV-associated dementia, compared to those who never smoked [25]. However, during the pre-ART era researchers were challenged with loss of follow-ups, misdiagnosis of dementia, and premature mortality, before the onset of dementia that could skew the results. The potential effect of smoking on the course of HIV-associated cognitive impairment in the ART era has received even less attention. A paper leaded by Wojna [26] is the only recent one, and the authors observed no associations between cognitive impairment and either current or past history of smoking. However, the study was cross-sectional, and the numbers of subjects and domains were limited, severely restricting the generalizability of results [25-26]. In summary, while prior studies have provided us with valuable information, multiple methodological factors restrict the generalizability of the results, such as participant sampling, measures of cognition, rating systems and limited follow-ups. Prior research has been mostly carried out on young and middle age subjects, with limited or no participation of older individuals [25-26]. These are substantial limitations when considering that they are becoming a sizable proportion of the patient population, and that current knowledge in this area is quite limited. Moreover, these differences in population and methods, along with survival times, pose challenges in the interpretation and applicability of available data. Thus, the relationship between smoking and cognitive impairment remains disproved.

As it is now established, the aging brain is more vulnerable to endogenous and exogenous insults, and we can expect staggering increases in HAND associated with smoking. In addition, our more recent analyses indicated that mentholated cigarettes have the potential to affect neurotrophic and neurotransmitters. Importantly, smoking can sustain an inflammatory response that could be highly deleterious for the CNS [18-19]. These findings lead to concern because cognitive deficits are likely to impair adherence to complex medical regimens, reduce tobacco cessation efforts and quality of life.

In summary, HIV, aging and smoking can collectively increase HAND rates around the world, creating a public health problem for which most nations are not prepared. Therefore, there is an urgent need for safe, effective, and affordable intervention approaches.

\section{HAART Benefits Hurt by Smoking}

Although HAART has helped to reduce opportunistic infection, morbidity and mortality rates are still higher than those observed in the general population. Given the rates of smoking among PLWH and the myriad of negative health effects induced by tobacco, scientists need to wonder if smoking could be a driving force. The answer is a strong "yes"; over the years our group, along with others, has documented marked increase in rates of respiratory infections as a result of tobacco associated immune impairments. Our studies identified an increased risk of smokers to develop Pneumocystis carinii pneumonia (PCP), more community acquired pneumonia and increased risk of developing Mycobacterium avium complex (MAC), a life-threatening infection affecting as near as half of PLWH [26-33]. These results were not unexpected given that MAC has been isolated in cigarettes. Since immunodeficiency and AIDS-related pulmonary infections have been associated with increased risks of developing maliganancies, it is not unexpected that in the Swiss HIV Cohort Study (SHCS) confirmed a strong association of smoking with lung cancer" (OR for current vs. never $=14.4)[34]$.

Other adverse events that have been found associated with tobacco are kidney and cardiovascular diseases. Chronic Kidney Disease (CKD) is not only turning to be an epidemic, it is one of the most costly illnesses. This is disturbing, as our findings suggest that approximately one out of every three CKD cases in PLWH could have been avoided if no one in the population had smoked [35]. With the advent of HAART, cardiovascular diseases have also become an issue of concern. The physiopathology behind the increased risk of cardiovascular disease in PLWH is still unclear, but epidemiological and clinical studies have extensively blamed certain antiretrovirals for these increasing odds. Notably, analyses from one of the multicenter cohorts (DAD Study Group) demonstrated that smoking is associated with greater risk of myocardial infarction than protease inhibitor therapy (relative risk, 2.83 vs. 1.2) [36]. These findings further underscore the need of more cessation strategies tailored for PLWH.

As a result, smokers have more new admissions and readmissions resulting in an excess cost of half a million dollar in hospital days. However, at the time we performed those analyses weight status was not available to gather additional conclusions. Besides, by contrasting prior expectations that smoking will be associated with normal to low body mass index, we have found that the use of mentholated cigarettes was associated with overweight and obesity. Therefore, additional analyses will be on the way to assess the combined effects of these interconnected risks.

\section{Missing Pieces of Critical Information: The Plausible Effects of the Widespread use of Mentholated Cigarettes among People Living with HIV}

Although $27 \%$ of U.S. current smokers report to mainly use mentholated cigarettes, also known as menthols, among PLWH our 
Citation: Miguez MJ (2012) Current Issues in Cigarette Smoking Among Persons Living with HIVIAIDS: A Growing Public Health Problem Surrounded by Missing Information and Misconceptions. J AIDS Clinic Res 3:e109. doi:10.4172/2155-6113.1000e109

Page 3 of 4

analyses indicated that a stunning $70 \%$ of the population use menthols [2]. Given the large number of menthol cigarette users, the first question that emerged is whether menthol cigarette smokers are exposed to more tobacco smoke toxins than smokers of non-menthol cigarettes. To answer this question we analyzed data of the FILTERS ongoing study and found that cotinine levels are higher when normalized for cigarettes smoked per day in menthol users than non-menthol smokers. Analyses by race yield the same conclusion: higher serum cotinine levels among menthol smokers in both African-Americans compared to whites. These findings are in accord with data yield from the general population and with tobacco industry documents, indicating that menthol enables deeper inhalation and may alter the frequency or volume of inhalation patterns.

In this framework, it will be worthwhile to investigate if smokers of menthol cigarettes have increased risk for diseases in comparison with smokers of non-menthol cigarettes? The answer to this question among PLWH is unknown, and is the focus of our current research efforts. Unfortunately, available data from the general population is very limited; only five epidemiological studies address lung cancer and lesser numbers were identified for other health outcomes [35-36]. None of them were specifically designed to address this scientific question, and results are contradictory. Accordingly, FDA has determined that evidence is insufficient to conclude that menthol users face a different risk of tobacco-caused diseases than smokers of non-menthol cigarettes, and therefore they do not recommend menthols to be banned [36].

Unfortunately, the lack of evidence seems to be hurting both the health and the national efforts to reduce smoking rates. Our study, along with those of others, indicated that PLWH had perceptions that are inconsistent with the motivation to quit smoking. Many expressed no health concerns, and argument that there are no studies showing otherwise. Many even talked about the relative harmfulness of menthols. In addition to these misperceptions, PLWH perceived benefits to smoking such as helping to cope with HIV, the potential of smoking for increasing CD4 cell counts and to help them maintain their weight. In summary, it is evident that old studies not applicable to the HAART era, the lack of new information and/or of public campaigns informing the public about tobacco plausible health risks are and will be harming the success achieved with HAART.

\section{Conclusions}

Early in the epidemic, preventive care was the cornerstone of HIV management; subsequently, the emergence of HAART shifted management and priorities to focus on complex antiretroviral regimens and increased adherence as the foundation of HIV care. Over the past years, it has become evident that to successfully manage a chronic disease we need to re-evaluate old myths and emphasize prevention. The scope and depth of the cigarette smoking problem underscores the importance of a new strategy to reduce the death and disability caused by the use of tobacco products. Researchers and public health professionals ought to address the plausible effects of mentholated cigarettes to ensure that PLWH are not at any additional harm. That strategy may develop out of a recent public debate about tobacco control policies that has intensified over the past three years. Current understanding of the plausible effects of smoking, menthols, and even smokeless tobacco suggests that there is a long way to go to protect our nation from more harm. Given that minorities are large consumers of menthols, perhaps we need to reflect more on the long-term consequences of menthols as a proximal factor rather than on more distant elements such as social inequalities.

\section{References}

1. http://www. Who.int/tobacco/en

2. Department of Health and Human Services (2010) Ending the Tobacco Epidemic: A Tobacco Control Strategic Action Plan for the U.S. Department of Health and Human Services. Washington, DC: Office of the Assistant Secretary for Health

3. Míguez-Burbano M, Shor-Posner G, Perez E, Ashkin D, Rodriguez A, et al (2004) Tobacco use: the epidemic striking HIV-infected subjects. HIV AIDS Rev 3: 26-32.

4. Lifson AR, Lando HA (2012) Smoking and HIV: Prevalence, Health Risks, and Cessation Strategies. Curr HIVIAIDS Rep 9: 223-230.

5. Tesoriero JM, Gieryic SM, Carrascal A, Lavigne HE (2010) Smoking among HIV positive New Yorkers: prevalence, frequency, and opportunities for cessation. AIDS Behav 14: 824-835

6. Centers for Disease Control and Prevention (2012) HIV Surveillance Report.

7. Moore RD, Chaisson RE (1999) Natural history of HIV infection in the era of combination antiretroviral therapy. AIDS 13: 1933-1942.

8. Crum-Cianflone NF, Grandits G, Echols S, Ganesan A, Landrum M, et al. (2010) Trends and Causes of Hospitalizations among HIV-infected Persons during the Late HAART Era: What is the Impact of CD4 Counts and HAART Use? J Acquir Immune Defic Syndr 54: 248-257.

9. Furber AS, Maheswaran R, Newell JN, Carroll C (2007) Is smoking tobacco an independent risk factor for HIV infection and progression to AIDS? A systemic review. Sex Transm Infect. 83: 41-46.

10. Marshall MM, McCormack MC, Kirk GD (2009) Effect of Cigarette Smoking on HIV Acquisition, Progression, and Mortality. AIDS Educ Prev 21: 28-39.

11. Paintsil E, Ghebremichael M, Romano S, Andiman WA (2008) Absolute CD4+ T-Lymphocyte Count as a Surrogate Marker of Pediatric Human immunodeficiency virus Disease Progression. Pediatr Infect Dis J 27: 629-635.

12. Francus T, Francus $Y$, Weksler ME, Becker CG (1987) Effects of tobacco glycoprotein (TGP) on the immune system. III. The effect of aging on the mitogenic response of human peripheral blood lymphocytes to TGP. Cell Immunol 105: 1-8.

13. Feldman JG, Minkoff H, Schneider MF, Gange SJ, Cohen M, et al. (2006) Association of cigarette smoking with HIV prognosis among women in the HAART era: a report from the Women's Interagency HIV Study. Am J Public Health 96: 1060-1065.

14. Kuller LH (2008) Elevated levels of IL-6 and D-dimer are associated with increased risk of death in patients with HIV. PLos Medicine 5: e203.

15. SMART Study Group, El-Sadr WM, Grund B, Neuhaus J, Babiker A, et al (2008) Risk for Opportunistic Disease and Death after Reinitiating Continuous Antiretroviral Therapy in patients with HIV previously receiving episodic therapy: a randomized trial. Ann Intern Med 149: 289-299.

16. Levin J (2009) HIV \& HAART Interruption Increase Inflammation and Disease. 16th CROI Conference on Retroviruses and Opportunistic Infections Montreal, Canada.

17. Amirayan-Chevillard N, Tissot-Dupont $\mathrm{H}$, Capo $\mathrm{C}$, Brunet $\mathrm{C}$, Dignat-George $\mathrm{F}$ et al. (2000) Impact of highly active anti-retroviral therapy (HAART) on cytokine production and monocyte subsets in HIV $\square$ infected patients. Clin Exp Immunol 120: $107-112$.

18. Appay V, Sauce D (2008) Immune activation and inflammation in HIV-1 infection: causes and consequences. J Pathol 214: 231-241.

19. Highleyman L (2010) Inflammation, Immune Activation and HIV

20. Neuhaus J, Jacobs Jr DR, Baker JV, Calmy A, Duprez D, et al. (2010) Markers of inflammation, coagulation, and renal function are ewlevated in adults with HIV-infection. J Infect Dis 201.

21. Shepp D. Chronic Inflammation \& Medical IIIness in HIV.

22. Míguez-Burbano MJ, Rosenberg R, Malow R, Burbano X, Devieux J, et al. (2010) The effect of mentholated cigarette use on interleukin- 6 responses across different HIV positive race/ethnic subgroups. Int $\mathrm{J}$ Infereron Cytokine Mediator Res. 2: 157-164.

23. Heaton R (2010) Persistence and progression of HIV-associated neurocognitive 
Citation: Miguez MJ (2012) Current Issues in Cigarette Smoking Among Persons Living with HIVIAIDS: A Growing Public Health Problem Surrounded by Missing Information and Misconceptions. J AIDS Clinic Res 3:e109. doi:10.4172/2155-6113.1000e109

impairment $(\mathrm{NCl})$ in the era of combination antiretroviral therapy (CART) and the role of comorbidities: the CHARTER study. Neurology 75: 20872.

24. Cohen R (2009) The Changing Face of HIV-Associated Cognitive and Neuropsychiatric Disturbance. Current Clinical Neurology 133: 1-54.

25. Burns DN, Hillman D, Neaton JD, Sherer R, Mitchell T, et al. (1996) Cigarette smoking, bacterial pneumonia, and other clinical outcomes in HIV-1 infection. Terry Beirn Community Programs for Clinical Research on AIDS. J Acquir Immune Defic Syndr Hum Retrovirol 13: 374-383.

26. Wojna V, Robles L, Skolasky RL, Mayo R, Selnes O, et al. (2007) Associations of cigarette smoking with viral immune and cognitive function in human immunodeficiency virus-seropositive women. J Neurovirol 13: 561-568.

27. Miguez-Burbano MJ, Ashkin D, Rodriguez A, Duncan R, Pitchenik A, et al. (2005) Increased risk of Pneumocystis Carinii and community-acquired pneumonia with tobacco use in HIV disease. Int J Infect Dis 9: 208-217.

28. Miguez-Burbano MJ, Shor-Posner G, Hadrigan S (2005) Non-tuberculous mycobacteria in HIV- infected patients: geographic, behavioural, and immunological factors. Lancet 5: 394-395.

29. Miguez-Burbano MJ, Burbano X, Ashkin D, Pitchenik A, Allan R, et al. (2003) Impact of tobacco use on the development of opportunistic respiratory infections in HIV seropositive patients on antiretroviral therapy. Addict Biol 8: 39-43.
30. Gordin FM, Roediger MP, Girard PM, Lundgren JD, Miro JM, et al. (2008) Pneumonia in HIV-infected persons: increased risk with cigarette smoking and treatment interruption. Am J Respir Crit Care Med 178: 630-636.

31. Rahmanian S, Wewers ME, Koletar S, Reynolds N, Ferketich A, et al. (2011) Cigarette Smoking in the HIV-Infected Population. Proc Am Thorac Soc 8: 313 319

32. Clifford GM, Lise M, Franceschi S, Egger M, Bouchardy C, et al. (2012) Lung cancer in the Swiss HIV Cohort Study: role of smoking, immunodeficiency and pulmonary infection. Br J Cancer 106: 447-452.

33. Míguez-Burbano MJ, Wyatt C, Lewis JE, Rodríguez A, Duncan R (2010) Ignoring the Obvious Missing Piece of Chronic kidney Disease in HIV: Cigarette Smoking. J Assoc Nurses AIDS Care 21: 16-24.

34. DAD Study Group, Friis-Møller N, Reiss P, Sabin CA, Weber R, et al. (2007) Class of antiretroviral drugs and the risk of myocardial infarction. N Engl J Med 356: 1723-1735

35. Mendiondo MS, Alexander LA, Crawford T (2010) Health profile differences for menthol and non-menthol smokers: findings from the National Health Interview Survey. Addiction 105: 124-140.

36. http://www.fda.gov/downloads/AdvisoryCommittees/ CommitteesMeetingMaterials/TobaccoProductsScientificAdvisoryCommittee/ UCM244977.pdf 